\title{
The effects of prehabilitation versus usual care to reduce postoperative complications in high-risk patients with colorectal cancer or dysplasia scheduled for elective colorectal resection: study protocol of a randomized controlled trial
}

Annefleur E. M. Berkel ${ }^{1 *}$, Bart C. Bongers², Marie-Janne S. van Kamp ${ }^{1}$, Hayke Kotte $^{3}$, Paul Weltevreden ${ }^{4}$, Frans H. C. de Jongh ${ }^{5}$, Michiel M. M. Eijsvogel ${ }^{5}$, A. N. Machteld Wymenga ${ }^{6}$, Marloes Bigirwamungu-Bargeman", Job van der Palen ${ }^{8}$, Marc J. van Det ${ }^{9}$, Nico L. U. van Meeteren ${ }^{2,10}$ and Joost M. Klaase ${ }^{1}$

\begin{abstract}
Background: Of all older patients that opt for elective colorectal surgery, approximately one-third has one or more postoperative complications, particularly those patients with a low cardiorespiratory fitness (ventilatory anaerobic threshold (VAT) $<11 \mathrm{~mL} / \mathrm{kg} / \mathrm{min}$ ). A physical exercise training program prior to surgery (prehabilitation) can improve their cardiorespiratory fitness. It remains to be seen whether prehabilitation also reduces postoperative complications, as most of the studies so far were rather underpowered, heterogeneous, and biased toward selection of patients with a lower risk of postoperative complications. The primary objective of this study is to evaluate the effects of a three-week prehabilitation program on 30-day postoperative complications in patients with a VAT $<11 \mathrm{~mL} / \mathrm{kg} / \mathrm{min}$ planned for elective colorectal resection for colorectal cancer or dysplasia.

Methods: In this multicenter prospective randomized controlled trial, patients $\geq 60$ years with colorectal cancer or dysplasia grade I, II, or III, planned for elective colorectal resection in two hospitals in the Netherlands, will be recruited. Eligible patients must have a score $\leq 7$ metabolic equivalents on the veterans-specific activity questionnaire, and should be able to perform a cardiopulmonary exercise test. A total of 86 patients will be randomized (block-stratified randomization) to prehabilitation (intervention group) or usual care (control group). For final inclusion, VAT should be $<11 \mathrm{~mL} / \mathrm{kg} / \mathrm{min}$. Three times a week for 3 weeks, a 60-min supervised prehabilitation session will be completed in community physical therapy practices by the 43 patients in the prehabilitation group, consisting of moderate-to-high intensity interval training to improve cardiorespiratory fitness, and resistance training to improve peripheral muscle strength. Additionally, patients perform home exercises twice a week on a moderate intensity level. The 43 patients in the usual care group will receive usual care.

(Continued on next page)
\end{abstract}

\footnotetext{
*Correspondence: a.berkel@mst.nl

'Department of Surgery, Medisch Spectrum Twente, PO Box 50 000, 7500,

KA, Enschede, The Netherlands

Full list of author information is available at the end of the article
} 
(Continued from previous page)

Discussion: Optimizing preoperative physical fitness may decrease the postoperative complication rate, may lead to fewer reoperations, less intense clinical care, a shorter length of stay, a more effective surgical planning (processoptimization), fewer readmissions, less intense rehabilitation, shorter rehabilitation period, earlier resumption of work, enhance patient perceived health-related quality of life, and promote performance in daily life. Costeffectiveness should therefore be expected and evaluated.

Trial registration: Medical Ethics Committee Twente, Enschede, the Netherlands (NL45001.044.13, September 3, 2013); Netherlands Trial Register (NTR; NTR4032, June 14, 2013).

Keywords: Colorectal surgery, Cardiorespiratory fitness, Ventilatory anaerobic threshold, Prehabilitation, Physical therapy, Exercise training, Postoperative complications, Physical functioning/fitness

\section{Background}

Worldwide, colorectal cancer is the third most common type of cancer for men and women [1]. In the Netherlands, about 15.000 people are diagnosed with colorectal cancer each year [2]. Surgery is the cornerstone of treatment in patients with colorectal cancer. However, the 30-day complication rate after elective colorectal resection is approximately one-third [3], including for example an anastomotic leakage, ileus, or wound infection. Colorectal cancer primarily occurs in the elderly. The level of psychophysiological reserve capacity (resilience) and comorbidities affect the tolerance to surgery in older patients [4]. According to the Dutch Surgical Colorectal Audit, approximately 35\% of the patients with colorectal cancer are aged between 60 and 70 years, and $40-50 \%$ of the patients are aged over 70 years [5]. These older patients more often have a low cardiorespiratory fitness.

In the literature, preoperative cardiorespiratory fitness has consistently been reported to be associated with postoperative outcome in major elective intra-abdominal surgery (e.g., morbidity, mortality, and length of stay) [6]. Cardiorespiratory fitness can be measured objectively using a cardiopulmonary exercise test (CPET) by determining the ventilatory anaerobic threshold (VAT). The VAT is an accurate and repeatable measurement [7] that can be obtained from the CPET, without a learning effect [8]. It is a physiological construct that occurs at submaximal exercise intensity. Hence, the VAT is only marginally influenced by the patient's ability and motivation to deliver a maximal effort. Previous studies showed that there is a significant association between a low VAT and postoperative complications in major non-cardiac surgery [8-21]. Patients with a VAT $<11 \mathrm{~mL} / \mathrm{kg} / \mathrm{min}$ have a higher likelihood for postoperative complications [17, 22].

The literature shows that high-risk patients (those patients with a higher likelihood of postoperative complications), who participated in a physical exercise training program prior to elective surgery (prehabilitation), improved their cardiorespiratory fitness [23-25], which has led to the hypothesis that postoperative complications can be reduced by prehabilitation [23,
25-27]. Dunne et al. [28] designed a four-week highintensity interval training program based on the work rate at the VAT for patients prior to hepatic resection. Their aim was to assess feasibility and to improve the preoperative VAT by $1.5 \mathrm{~mL} / \mathrm{kg} / \mathrm{min}$ (a $10 \%$ increase). They found their program to be feasible in sedentary healthy volunteers, resulting in a $>10 \%$ improvement in cardiorespiratory fitness (VAT). Using preoperative risk stratification, a $10 \%$ improvement in cardiorespiratory fitness in patients undergoing hepatic resection would shift $30 \%$ of the high-risk patient group to a low-risk patient group [28].

Prehabilitation programs may have beneficial effects on postoperative outcome; however, the effects on physical functioning, morbidity, mortality, and length of stay are inconclusive. In a systematic review of Moran et al. [6] that aimed to assess the ability of prehabilitation to influence postoperative outcome after intra-abdominal surgery, it was concluded that prehabilitation appears to be beneficial in decreasing the incidence of postoperative complications. Additionally, a systematic review of Bruns et al. [29] reported that prehabilitation can improve the physical fitness of older patients undergoing colorectal surgery; however, no significant effect on the reduction of complications or length of stay could be demonstrated. They concluded that previous studies on the effects of prehabilitation in patients undergoing elective colorectal resection provided no clear and complete description of the content and execution of the prehabilitation program, and that in most of these studies no adequate sample size calculation was performed [29]. Hijazi et al. [30] recently performed a systematic review of all comparative studies on prehabilitation versus usual care in patients undergoing abdominal cancer surgery. No significant difference was found in postoperative complications between prehabilitation and usual care groups. They concluded that prehabilitation programs for patients undergoing major abdominal cancer surgery remain heterogeneous in terms of their composition, duration, mode of administration, compliance, and outcomes measures used to quantify their impact. Therefore, the findings and recommendations 
are limited. They recommended for future research to standardize these aspects prior to the evaluation of the effects of prehabilitation programs on a larger scale [30]. Thus, it remains to be demonstrated whether a prehabilitation program reduces postoperative complications [31]. Previous studies are biased toward selection of patients with low risk of postoperative complications, and it is unlikely that a prehabilitation program of a few weeks will give a clinically relevant improvement of cardiorespiratory fitness in these low-risk patients.

In summary, current studies addressing the effects of prehabilitation on overall postoperative complications in patients with colorectal cancer are inconclusive, opposing, and of low-to-moderate methodological quality and/or therapeutic validity (concept of Hoogeboom et al. [32]). More high-quality studies are needed to validate its use in the preoperative setting $[6,33]$. Recently though, it has been demonstrated that personalized prehabilitation reduced the number of patients with postoperative complications by $51 \%$ in high-risk patients undergoing elective major abdominal surgery [24]. Nevertheless, the selection of high-risk patients in this study of Barberan-Garcia et al. [24] was not performed by the use of an objective test, such as a preoperative CPET. Based on the previous published work, the current study will be different in several ways: 1) we perform a validated procedure of preoperative risk stratification with each eligible patient, in order to include only patients with a higher risk for postoperative complications based on cardiorespiratory fitness, 2) we preoperatively measure cardiorespiratory fitness objectively by using an evidence-based CPET to assess the VAT for risk stratification, 3) we design a personalized prehabilitation program based on the results of the CPET, 4) we train at a physical therapy practice close to the patient's home and not at the outpatient clinic of the hospital, as high-risk patients and their informal caregivers are less or even unable and/or less motivated to visit the hospital to train three times a week during the preoperative period, 5) we give an exact description of the content and execution of the prehabilitation program, 6) we select a patient group with a homogeneous diagnosis, that is patients with colorectal cancer or dysplasia only, and 7) we performed an adequate sample size calculation based on previous data on the effect of prehabilitation in patients undergoing elective hepatic resection [25, 28], as well as on the reported complication rates in frail patients undergoing colorectal resection presented by Robinson et al. [34].

The primary objective of this randomized controlled trial is to evaluate the hypothesis that a three-week prehabilitation program (intervention group) will reduce the number of postoperative complications from $50 \%$ to $20 \%$ in patients with a preoperative VAT $<11 \mathrm{~mL} / \mathrm{kg} / \mathrm{min}$ who will undergo elective colorectal resection for colorectal cancer or dysplasia grade I, II, or III, when compared to usual care (control group).
The secondary objectives are 1) to observe whether changes in preoperative cardiorespiratory fitness occur in the intervention group (prehabilitation, single-arm study design), 2) to evaluate the effect of the prehabilitation program on length of stay, 3) to examine whether patients with a preoperative VAT $\geq 11 \mathrm{~mL} / \mathrm{kg} / \mathrm{min}$, and who will therefore not be included in the randomized controlled trial, have fewer postoperative complications than patients in the prehabilitation or usual care group (for the prehabilitation group, we will use the postprehabilitation CPET data), 4) to investigate the value of a limited geriatric assessment in this patient group to perform preoperative risk stratification, and 5) to evaluate the cost-effectiveness of prehabilitation in high-risk patients by performing a cost-effectiveness analysis.

\section{Methods}

\section{Study design}

This study is a multicenter prospective randomized controlled trial. The trial has started in February 2014 and will run till patient inclusion is completed (probably at the end of 2018) at Medisch Spectrum Twente in Enschede and Ziekenhuisgroep Twente in Almelo, two large community teaching hospitals in the eastern part of the Netherlands. In this manuscript, the latest version of the study protocol (version 9, March 2017) is presented. The study is approved by the Medical Ethics Committee Twente in Enschede, the Netherlands (registration number P13-18), and is registered in the Netherlands Trial Register (NTR4032). Protocol amendments will be agreed and approved by the Medical Ethics Committee Twente.

\section{Participants}

To be eligible to participate in this study, a patient must meet all of the following inclusion criteria: 1) $\geq 60$ years, 2) colorectal cancer or premalignant colorectal lesions (dysplasia grade I, II, or III) requiring colorectal resection, 3) undergoing elective colorectal resection at Medisch Spectrum Twente, Enschede, or at Ziekenhuisgroep Twente, Almelo, 4) having a life expectancy of more than 6 months as estimated by the surgeon, 5) has given informed consent to participate in this study, 6) a metabolic equivalents of task (MET) score $\leq 7$ on the veterans-specific activity questionnaire (VSAQ), 7) able to perform a CPET, 8) a VAT $<11 \mathrm{~mL} / \mathrm{kg} / \mathrm{min}$ as measured at the baseline CPET, and 9) willing to perform prehabilitation at a community physical therapy practice in the adherence area of both hospitals. All patients not meeting these criteria will not be considered for inclusion. Participants can leave the study at any time for any reason if they wish to do so, without any consequences. The investigator can decide to withdraw a participant from the study for urgent medical reasons. 


\section{Recruitment}

All patients will be identified at the multi-disciplinary oncology meetings, and will be evaluated at the outpatient clinic by the surgeon or surgical resident. As most postoperative complications are to be expected in patients with a low cardiorespiratory fitness [6], we aim to include only patients who are expected to be unfit in the pre-study assessments. To select patients with a potentially low cardiorespiratory fitness, all patients identified at the multi-disciplinary oncology meeting are asked to fill out the VSAQ [35]. The VSAQ links physical activities to a particular MET score, based on the Compendium of Physical Activities [36]. Snowden et al. [14] found that patients with a VSAQ score $>7$ METs are most likely not to have major postoperative complications. Therefore, we define a low perceived cardiorespiratory fitness as a VSAQ score $\leq 7$ METs.

Patients with a VSAQ score $\leq 7$ METs will receive very limited information about the study. They will be asked to participate in a study addressing the role of cardiorespiratory fitness (assessed with a CPET) on postoperative complications following colorectal resection. If informed consent is obtained (Additional file 1), the patient will be randomized to the intervention group (prehabilitation) or the control group (usual care), and they will undergo a CPET to obtain objective information about their cardiorespiratory fitness, as standard work-up. Eventually, patients with a VAT $<11 \mathrm{~mL} / \mathrm{kg} / \mathrm{min}$ can participate in the study, whereas randomized patients with a VAT $\geq 11 \mathrm{~mL} / \mathrm{kg} / \mathrm{min}$ cannot participate in the study (in the intervention group, nor in the usual care group). See Fig. 1 for a flow diagram of the study design.

Patients in the prehabilitation group and the usual care group will be informed differently. The patients in the prehabilitation group will be fully informed about the study in a patient information letter that explains the aims and expectations of this study, as well as the risks and benefits of participating. Patients in the usual care group will not receive information about the possible effects of prehabilitation to avoid the risk that these patients will initiate preoperative physical exercise training themselves. The usual care group will receive a patient information letter about the registration of perioperative data and the hypothesized relation of cardiorespiratory fitness with postoperative complications after colorectal resection. Patients in both the prehabilitation group and the usual care group will be contacted by the clinical research coordinator a few days later to answer their questions, and to inform them about the CPET date. This procedure is approved by the Medical Ethics Committee Twente.

\section{Randomization}

To analyze the effect of a three-week prehabilitation program on postoperative complications, patients with a
VSAQ score $\leq 7$ will be randomized in the intervention group (prehabilitation) or the control group (usual care) by using block-stratified randomization [37]. Randomization is stratified by disease and treatment type: 1) patients with colon cancer, 2) patients with rectal cancer who will receive one week of neoadjuvant radiotherapy, and 3) patients with rectal cancer who will receive five weeks of neoadjuvant chemoradiotherapy. The clinical research coordinator of the surgical department will be responsible for the randomization. Participants, care providers, and outcome assessors will not be blinded after randomization. However, the data analysts will be blinded to group allocation and are independent to the intervention. After randomization, all participants will perform a CPET for final inclusion, as merely patients with a VAT $<11 \mathrm{~mL} / \mathrm{kg} /$ min can participate in the study.

\section{Interventions}

\section{Prehabilitation program}

Patients in the prehabilitation group will participate in a three-week (three sessions per week, nine sessions in total) supervised physical exercise training program prior to colorectal resection. The prehabilitation program is based on the program developed by Dunne et al. $[25,28]$, which has been shown to be feasible in sedentary healthy volunteers [28], and later demonstrated to deliver improvements in CPET scores and perceived health-related quality of life prior to hepatic resection [25]. In the studies of Dunne et al. [25, 28] prehabilitation consisted of twelve interval training sessions over a four-week period, whereas in the present study patients will perform nine preoperative sessions of individuallytailored interval training (personalized prehabilitation) over a three-week period. In the literature, it has been shown that as little as six sessions of 'all-out' highintensity interval training over two weeks is sufficient to enhance cardiorespiratory fitness and improve skeletal muscle oxidative capacity in untrained and recreationally active individuals $[38,39]$. However, a comparison with our patients with colorectal cancer and a low cardiorespiratory fitness cannot be made, because their 'all-out' exercise program differs from our exercise intervention, as 'all-out' exercise may not be safe, tolerable, or appealing for our patient group.

Patients will prehabilitate on Monday, Wednesday, and Friday, or on Tuesday, Thursday, and Saturday to improve their physical fitness preoperatively. Each 60-minute training session consists of moderate-to-high intensity interval training to improve cardiorespiratory fitness, and resistance training to improve peripheral muscle strength (see Tables 1 and 2). The interval training program will be tailored to each individual patient, based on the results of the CPET. The training sessions will be supervised by a selected group of trained physical therapists in community 


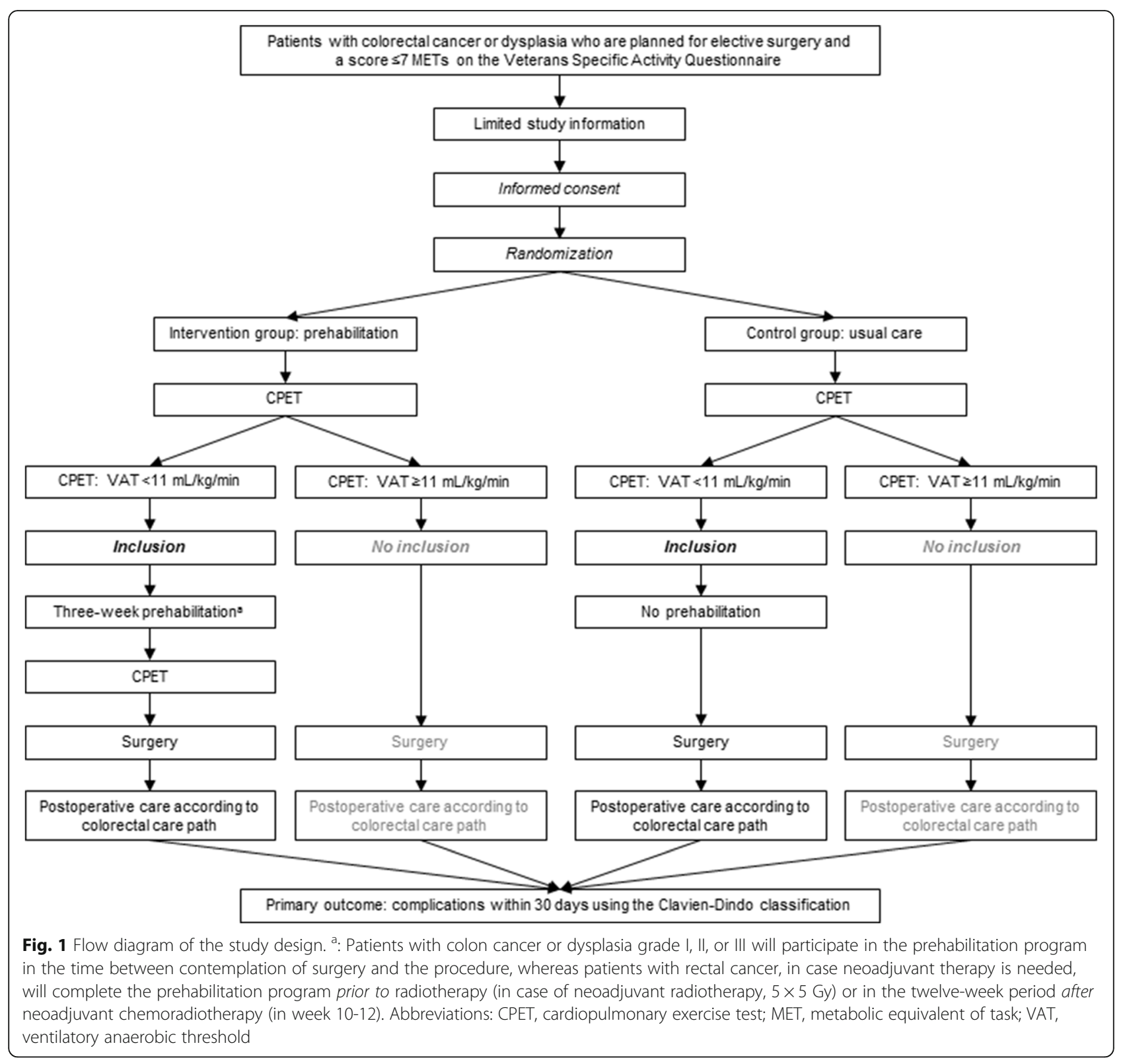

physical therapy practices in de adherence area of the hospitals. Additionally, the patient performs unsupervised home exercises at a moderate exercise intensity (e.g., walking, cycling, or stair climbing) twice a week for at least 30 minute, as checked by the physical therapist. We added these functional exercises to the prehabilitation program as de Vreede et al. [40] demonstrated that functional-task exercises are effective at improving functional task performance in healthy elderly women. Hence, home-based functional exercises that are of relevance for a patient may have an important role in helping patients to mobilize quickly postoperatively, to be physically active throughout the hospitalization period postoperatively, and to maintain independent physical functioning. Indeed, prehabilitation ideally should not focus merely on the preoperative period. Postoperatively, hospital culture and infrastructure should stimulate the patient to be physically active. However, in most hospitals health care is entirely organized around the patient's bed [41], which invites patients to lie in bed, even without a medical reason. Bed-centered care probably also hampers the effectiveness of prehabilitation to improve postoperative outcomes.

Patients with colon cancer or dysplasia will participate in the prehabilitation program in the time between contemplation of surgery and the procedure, whereas patients with rectal cancer, in case neoadjuvant therapy is needed, will complete the physical exercise training program prior to radiotherapy (in case of neoadjuvant 
Table 1 Content of the three-week interval training program

\begin{tabular}{|c|c|c|}
\hline & Duration & Intensity $^{a}$ \\
\hline Warm-up & $7 \mathrm{~min}$ & $50 \%$ \\
\hline Interval session & $30 \mathrm{~min}$ & $\begin{array}{l}\text { Work interval: } 120 \% \\
\text { Recovery interval: } \\
50 \%\end{array}$ \\
\hline $\begin{array}{l}\text { Week 1, } \\
\text { session 1, 2, and } 3\end{array}$ & $\begin{array}{l}\text { Work interval: } 120 \mathrm{~s} \\
\text { Recovery interval: } 180 \mathrm{~s}\end{array}$ & \\
\hline $\begin{array}{l}\text { Week 2, } \\
\text { session 4, 5, and } 6\end{array}$ & $\begin{array}{l}\text { Work interval: } 140 \mathrm{~s} \\
\text { Recovery interval: } 160 \mathrm{~s}\end{array}$ & \\
\hline $\begin{array}{l}\text { Week 3, } \\
\text { session } 7,8 \text {, and } 9\end{array}$ & $\begin{array}{l}\text { Work interval: } 160 \mathrm{~s} \\
\text { Recovery interval } 140 \mathrm{~s}\end{array}$ & \\
\hline Cool-down & $3 \mathrm{~min}$ & $60 \%$ \\
\hline
\end{tabular}

a: Expressed as a percentage of the individually achieved work rate (in Watt) at the ventilatory anaerobic threshold obtained from the cardiopulmonary exercise test

radiotherapy, $5 \times 5 \mathrm{~Gy}$ ) or in the twelve-week period after neoadjuvant chemoradiotherapy (training in the last three weeks before surgery).

\section{Usual care group}

Patients in the control group receive usual care and will not participate in a prehabilitation program. In addition, no advice about physical exercise training is offered to them.

\section{Measurements}

Both the prehabilitation group and usual care group will participate in a series of outcome measure assessments at baseline, seven days postoperatively, and 30 days postoperatively, whereas the prehabilitation group will participate in a series of outcome measure assessments after the three-week prehabilitation program as well (see Table 3).

Table 2 Content of the three-week peripheral resistance training program $^{a}$

\begin{tabular}{llll}
\hline & Repetitions $^{\text {b }}$ & Duration & Intensity $^{c}$ \\
\hline Week 1, session 1, 2, and 3 & $3 \times 8$ repetitions & 20 min & $70 \%$ of 1RM \\
Week 2, session 4, 5, and 6 & $3 \times 8$ repetitions & 20 min & $76 \%$ of 1RM \\
Week 3, session 7, 8, and 9 & $3 \times 8$ repetitions & 20 min & $82 \%$ of 1RM
\end{tabular}

${ }^{a}$ : Peripheral resistance training of the large muscle groups of the lower and upper extremities using open and closed kinetic chain exercises (without physical therapy equipment or machines): crouching by means of squat exercises (primary muscle group: quadriceps femoris; secondary muscle groups: gluteal muscles, hamstring muscles, and gastrocnemius muscle), pulling by means of pulley exercises (primary muscle groups: latissimus dorsi muscle and rhomboid muscles; secondary muscle groups: biceps brachii muscle, rotator cuff, and trapezius muscle), pushing by means of pulley exercises (primary muscle group: pectoral muscles; secondary muscle groups: shoulder muscles, triceps brachii muscle), and lifting by means of pulley exercises (primary muscle groups: trunk muscles and shoulder muscles; secondary muscle groups: quadriceps femoris, gluteal muscles, and trapezius muscle)

${ }^{\mathrm{b}}$ : Rest intervals of $60-90 \mathrm{~s}$ between each set of repetitions

: Based on the 10RM, the patient's 1RM was determined using the Oddvar Holten diagram

Abbreviations: 1RM, one-repetition maximum; 10RM, ten-repetition maximum

\section{Cardiopulmonary exercise test}

A CPET will be used at baseline to assess cardiorespiratory fitness, as indicated by the VAT, in order to select and thereupon advice/invite eligible patients to participate in the study. For patients in the prehabilitation group, a second CPET will be used to investigate the effect of the prehabilitation program on cardiorespiratory fitness (singlearm study design). Patients free from any absolute and/or relative exclusion criteria, as stated by the American Thoracic Society and American College of Chest Physicians position statement [42], will be able to perform the CPET. The following standardized pre-test instructions will be given to the patients: 1) consume the last (light) meal at least 2 hours before exercise testing, 2) adhere to usual use of medication, and 3) wear comfortable sporting clothes and shoes. The CPET will be performed under controlled conditions at the lung function department of both hospitals, using a calibrated electronically braked cycle ergometer (Ergoline, Ergoselect 100, Bitz, Germany at Medisch Spectrum Twente and Lode Excalibur Sport, Lode BV, Groningen, the Netherlands at Ziekenhuisgroep Twente). Seat height will be adjusted to the patient's leg length. During the CPET, patients breathe through a facemask (Hans Rudolph, Kansas City, MO, USA) connected to a Triple V volume transducer to perform breath-by-breath measurements of oxygen uptake, carbon dioxide production, respiratory flow, and volume parameters (Oxycon Pro, Jaeger, Hoechberg, Germany). In both hospitals the same respiratory gas analysis system will be used. Before each CPET at the Medisch Spectrum Twente and each morning at the Ziekenhuisgroep Twente, flow-volume calibration (three-liter syringe) and gas calibration (ambient air and a gas mixture of $16 \%$ oxygen and $5 \%$ carbon dioxide) will be performed, and ambient conditions are set. Twelve-lead electrocardiography will be used to continuously monitor heart rate during the test. Blood pressure (SunTech Tango +, SunTech Medical, Inc., Marrisville, NC, USA) will be automatically measured every three minutes to be aware of extreme hypertension or to detect hypotension. Oxygen saturation (Nonin 9600, Nonin Medical, Inc., Plymouth, $\mathrm{MN}, \mathrm{USA}$ ) is continuously recorded during the test by finger pulse oximetry.

After the test protocol is explained to the patient, the CPET starts with a two-minute rest period during which baseline cardiopulmonary data are collected. The patient then starts unloaded cycling at a pedaling frequency of 60-80 rotations per minute for three minutes, where after the work rate will be increased every minute by 5 to $15 \mathrm{~W}$, depending on the patient's physical fitness level and aimed at reaching a maximal effort within eight to twelve minutes [43]. The CPET continues until the patient is unable to maintain a pedaling frequency above 60 rotations per minute due to volitional exhaustion and despite strong verbal encouragement. The CPET will be 
Table 3 Schedule of enrolment, interventions, and assessments

\begin{tabular}{|c|c|c|c|c|c|c|c|c|c|}
\hline \multirow{3}{*}{$\begin{array}{l}\text { STUDY PERIOD } \\
\text { TIMEPOINT }\end{array}$} & \multirow{3}{*}{$\begin{array}{l}\text { Enrolment } \\
-\mathrm{t}_{1}\end{array}$} & \multirow{3}{*}{$\begin{array}{l}\text { Allocation } \\
0\end{array}$} & \multicolumn{5}{|c|}{ Post-allocation } & \multicolumn{2}{|c|}{ Follow-up } \\
\hline & & & \multirow[t]{2}{*}{ Pre-prehabilitation } & \multicolumn{3}{|c|}{ Prehabilitation } & \multirow[t]{2}{*}{ Post-prehabilitation } & & \\
\hline & & & & Week 1 & Week 2 & Week 3 & & Day 7 post-surgery & Day 30 post-surgery \\
\hline \multicolumn{10}{|l|}{ ENROLMENT: } \\
\hline Eligibility screening & $x$ & & & & & & & & \\
\hline VSAQ score (METs) & $x$ & & & & & & & & \\
\hline Informed consent & $x$ & & & & & & & & \\
\hline Allocation & & $x$ & & & & & & & \\
\hline CPET & $x$ & & & & & & & & \\
\hline \multicolumn{10}{|l|}{ INTERVENTIONS: } \\
\hline \multicolumn{10}{|l|}{$\begin{array}{l}\text { Intervention group } \\
\text { (prehabilitation } \\
\text { program) }\end{array}$} \\
\hline \multicolumn{10}{|l|}{ Usual care group } \\
\hline \multicolumn{10}{|l|}{ ASSESSMENTS: } \\
\hline Baseline characteristics & $x$ & & & & & & & & \\
\hline SNAQ score (0-5) & $x$ & & & & & & & & \\
\hline $\begin{array}{l}\text { VAT on CPET } \\
(\mathrm{mL} / \mathrm{kg} / \mathrm{min})\end{array}$ & & & $x$ & & & & $x^{a}$ & & \\
\hline TUG test (s) & & & $x$ & & & & $x^{a}$ & & \\
\hline Muscle strength test & & & $x$ & & & & $x^{a}$ & & \\
\hline IADL & & & $x$ & & & & & & $x$ \\
\hline GDS 15 & & & $x$ & & & & & & $x$ \\
\hline EORTC QLQ-C30 & & & $x$ & & & & & & \\
\hline GFI & & & $x$ & & & & & & $x$ \\
\hline $\begin{array}{l}\text { Brief illness perception } \\
\text { questionnaire }\end{array}$ & & & $x$ & & & & $x^{a}$ & & \\
\hline EQ-5D & & & $x$ & & & & $x^{a}$ & & \\
\hline $\begin{array}{l}\text { Postoperative } \\
\text { complications }\end{array}$ & & & & & & & & $x$ & $x$ \\
\hline
\end{tabular}

${ }^{a}$ : Only for patients in the prehabilitation group

Abbreviations: CPET, cardiopulmonary exercise test; EORTC QLQ-C30, quality of life questionnaire of the European organisation for research and treatment of cancer; EQ-5D, EuroQol 5D; GDS 15, geriatric depression scale 15; GFI, Groningen frailty indicator; IADL, instrumental activities of daily living; MET, metabolic equivalent of task; SNAQ, short nutritional assessment questionnaire; TUG, timed up-and-go; VAT, ventilatory anaerobic threshold; VSAQ, veterans-specific activity questionnaire

considered to be at or near the maximal level when the patient shows clinical signs of intense effort (e.g., unsteady biking, sweating, and clear unwillingness to continue exercising despite strong encouragement), when the patient is unable to maintain a pedaling frequency above 60 rotations per minute, and when at least one of the following criteria is met: a heart rate at peak exercise of $>95 \%$ of predicted (predicted peak heart rate [beats $/ \mathrm{min}$ ] $=208-0.7 \times$ age $[$ years] $)$ or a respiratory exchange ratio at peak exercise of $>1.10$. After termination of the CPET, the patient will complete a recovery phase of unloaded cycling at a low pedaling frequency for five minutes. A clear description of adverse events and early test termination will be given.

During the CPET, absolute values at peak exercise are calculated as the average value over the last 30 seconds prior to termination of the test. Peak heart rate is defined as the highest heart rate achieved during the CPET. Cardiorespiratory fitness will be assessed by determining the VAT, by evaluating the peak oxygen uptake in case the patient performed a maximal effort, and by calculating the oxygen uptake efficiency slope [44]. The VAT will be detected by using the V-slope method, which is based on analyzing the slopes of oxygen uptake and carbon dioxide production. The VAT can be determined by using computerized regression analysis of these slopes. The point at which the linear slope of the relation between the carbon dioxide production and oxygen uptake changes, is called the VAT [45]. When the V-slope is expected to be unreliable, the ventilatory equivalents will be used to determine the VAT [46]. In this ventilatory equivalents method, the VAT will be defined as the point at which the ventilatory equivalent for oxygen and the partial end-tidal oxygen tension reached a minimum and thereafter began to rise in a consistent manner, coinciding with an unchanged ventilatory equivalent for carbon dioxide and partial end-tidal carbon dioxide tension [42]. The inter-observer variability in a preoperatively measured VAT has been reported to be acceptable for experienced clinicians [7], and DeCato et al. [47] recently reported short-term repeatability for CPET parameters in 
healthy participants in a similar test protocol, and they found no evidence for a learning effect and no significant differences in variability related to sex, age, fitness level, and diurnal factors.

\section{Muscle strength}

To investigate the effect of the prehabilitation program on muscle strength, a trained physical therapist will measure handgrip strength and quadriceps strength at baseline for both groups and after three weeks of prehabilitation for the prehabilitation group.

Handgrip strength (recorded in kilograms) will be assessed using the Jamar dynamometer (Sammons Preston, Rolyon, Bolingbrook, IL, USA) [48]. Handgrip strength has shown to be an indicator of skeletal muscle mass and a predictor of the risk of postoperative complications $[49,50]$. The test will be performed in upright position with the elbows stretched in a straight line downwards, starting with the dominant hand. First, the handle position, in which the patient has maximum strength, will be determined. Patients will be asked to squeeze the handle as forcefully as possible for about two seconds. Hereafter, two more measurements will be performed (three grip measurements per hand). Each measurement will be followed by a pause of 15-20 seconds. Mathiowetz [48] compared the Jamar and Rolyan hydraulic dynamometers for measuring grip strength, and showed that the Jamar and Rolyan dynamometers have acceptable concurrent validity with known weights, excellent inter-instrument reliability, and strong concurrent validity.

Quadriceps strength (recorded in Newton) will be measured with a hand-held dynamometer (MicroFET2, Hoggan Health Industries Inc., West Jordan, UT, USA), which has been shown to be a feasible, inexpensive, and portable test of the quadriceps muscle strength for use in healthy older people [51]. Patients will be seated at the examination table with their lower limbs bent over the edge (knees and hips flexed at $90^{\circ}$ ). If necessary, additional stabilization will be provided at the patients' shoulders by a second examiner. The test will be performed three times per leg, starting with the dominant leg. The hand-held dynamometer will be placed on the anterior aspect of the tibia at the level of the malleoli. The physical therapist will give resistance in the opposite direction. The patient must hold the leg in place. The resistance will gradually be increased in six seconds till maximum resistance/strength. The measurement will be stopped when the patient moves his leg. Each measurement will be followed by a pause of 30 seconds.

\section{Functional mobility}

To test (changes in) functional mobility, the physical therapist will execute the timed up-and-go (TUG) test at baseline for both groups and after three weeks of training for the prehabilitation group. The TUG test, a reliable and valid test, measures the time (recorded in seconds) a patient needs to stand up from a standard arm chair (approximate seat height of $46 \mathrm{~cm}$ ), walk three meters, turn, walk back to the chair, and sit down again $[52,53]$. The patient will perform the test once before being timed, in order to become familiar with the test and therefore minimize the learning effect.

In the literature, there are a few studies that demonstrated the use of the TUG test to identify patients at risk for impaired postoperative outcome [52, 54, 55]. Huisman et al. [55] showed that the TUG test was an independent predictor of the occurrence of major complications in a onco-geriatric surgical population. In this study, the predictive value of the TUG test on postoperative outcome will be determined. The TUG test is easy to perform, cheap, and does not need special equipment and might therefore be an alternative for the CPET.

\section{Nutritional status}

Assessment of nutritional status using the short nutritional assessment questionnaire (SNAQ) score [56] is part of usual care for our patients undergoing colorectal resection, and is evaluated at baseline for both the intervention and control group. The SNAQ is an easy, short, valid, and reproducible questionnaire for early detection of malnutrition [56]. Patients with a SNAQ score of 2 require nutritional support, whereas patients with a SNAQ score $\geq 3$ require supervision by a dietician and nutritional support. In this study the SNAQ score will be used as a baseline characteristic.

\section{American Society of Anesthesiologists score}

The American Society of Anesthesiologists (ASA) score is the most widely used system to describe a patient's preoperative health status. The ASA score has five classes ranging from class I, a completely healthy patient, to class V, a moribund patient who is not expected to live 24 hours with or without surgery $[57,58]$. The ASA score will be assessed at baseline for both groups.

\section{World Health Organization performance scale}

The World Health Organization (WHO) performance scale is a tool to assess how a patient's disease is progressing, how it affects activities of daily life, and it helps the clinician to determine appropriate treatment and prognosis [57]. The WHO performance scale will be assessed at baseline in the prehabilitation group and in the usual care group. 


\section{Charlson comorbidity index}

The Charlson comorbidity index $[59,60]$ will be scored at baseline for the prehabilitation group and usual care group. It can be a useful preoperative tool in predicting morbidity and mortality outcomes in patients with colorectal cancer [61].

\section{Questionnaires}

Every patient will visit an oncology nurse prior to colorectal resection, which is part of usual care. The oncology nurse will ask the patient to fill out several questionnaires: the instrumental activities of daily living (IADL) [62-64], geriatric depression scale 15 (GDS 15) $[65,66]$, the quality of life questionnaire of the European organization for research and treatment of cancer (EORTC QLQ-C30) [67, 68], and the Groningen frailty indicator (GFI) [69]. The IADL will investigate whether a patient experiences difficulties in carrying out instrumental activities that are essential to independent living. The GDS 15 will be used to measure perceived depressive symptoms or emotional health, and has good reliability, validity, sensitivity, and specificity for older people [70]. Patients will answer 'yes' or 'no' to fifteen questions depending on how they have felt over the past week. A score of 0-4 indicates normal mood or emotional health status, 5-9 indicates mild depression, and 10-15 indicates moderate-to-severe depression. The EORTC QLQ-C30 is a reliable and valid measure of the perceived health-related quality of life of patients diagnosed with cancer in multicultural clinical research settings [67]. The GFI is a short and easy-to-use instrument to measure frailty, which is defined as a loss of resources in several domains of functioning, which leads to a declining reserve capacity for dealing with stressors [69]. The patient will be asked to fill out the IADL, GDS 15, and GFI for the second time 30 days after surgery in order to investigate the effect of surgery on the IADL, depression, quality of life, and frailty, in both the prehabilitation and usual care group.

A limited geriatric assessment will be performed at baseline for the prehabilitation group and the usual care group, measuring comorbidity with the Charlson comorbidity index, signs of depression using the GDS 15, and functional status using the IADL. We will investigate the value of the limited geriatric assessment to predict postoperative complications. In addition, the GFI will be used in order to examine whether a screening tool can replace this limited geriatric assessment.

Illness perception will be recorded in both groups by using the brief illness perception questionnaire, a quick and easy to use questionnaire [71]. The face and content properties were found to be acceptable, and the reproducibility showed moderate-to-good reliability [71]. Illness perception is an important factor of health. Patients' personal thoughts about the symptoms they experience can be seen as one of the psychosocial factors by which variance in physical functioning in patients can be explained [71]. The physical therapist will ask patients in the prehabilitation group to fill out the brief illness perception questionnaire again after the three-week training period, in order to investigate the effect of the prehabilitation program on illness perception.

Health status will be evaluated by using the EuroQol 5D (EQ-5D) [72]. The EQ-5D questionnaire is a short profile measure of perceived health-related quality of life, and has five domains: 1) perceived mobility, 2) self-care, 3) usual activities, 4) pain/discomfort, and 5) anxiety/depression. Each domain is scored by patients according to three levels (no problems, some problems, extreme problems), for which quality weights (to enable calculation of quality adjusted life years for use in economic evaluations) are available [73]. The EQ-5D is widely used in oncology and it has shown a reasonable degree of reliability, content validity, construct validity, and responsiveness in the majority of available studies [74]. The physical therapist will ask patients in the prehabilitation group to fill out the EQ-5D again after the three-week training period, in order to investigate the effect of the prehabilitation program on health status.

\section{Study outcomes \\ Primary study parameter}

The primary study parameter is the number of overall postoperative complications within 30 days after surgery in the prehabilitation group and usual care group. Complications will be divided in surgical and nonsurgical complications. Surgical complications will be scored as anastomotic leakage, perineal wound complication, rectal stump abscess, intra-abdominal abscess, fistula, sepsis, ileus, abdominal wound complication, intestinal necrosis, stoma complication, urological complication, bleeding, iatrogenic intestinal injury, or iatrogenic vascular injury. Non-surgical complications will be scored as cardiovascular, pulmonary, thromboembolic, renal, or neurological. Complications will be recorded and graded by using the Clavien-Dindo classification $[75,76]$. Readmissions within 30 days will also be recorded.

\section{Secondary study parameters}

Secondary study parameters are 1) cardiorespiratory fitness, as indicated by the VAT, after the prehabilitation program in the intervention group just before surgery, 2) length of stay, 3) postoperative complications within 30 days after surgery in patients with a preoperative VAT $\geq 11 \mathrm{~mL} / \mathrm{kg} / \mathrm{min}$ and who will therefore not be included in the randomized controlled trial compared to 
patients in the prehabilitation or usual care group (for the prehabilitation group, we will use the postprehabilitation CPET data), 4) the value of a limited geriatric assessment in this patient group to predict (in combination with VAT) postoperative complications, and 5) cost-effectiveness of prehabilitation.

\section{Other study parameters}

Preoperative factors that could be associated with postoperative complications will be recorded, these include: age, sex, body height, body mass, body mass index, nutritional status, smoking status, location and type of the tumor, presence or absence of metastases, CPET parameters other than the VAT, WHO performance score, ASA score [77], Charlson comorbidity index [59], type of surgical resection, muscle strength, and the TUG test.

\section{Safety}

All adverse events reported spontaneously by participating patients, or observed by the investigator or his staff will be recorded.

\section{Data analysis}

\section{Sample size calculation}

Nowadays, there is 33\% 30-day morbidity and 3.8\% 30-day mortality in patients undergoing colorectal surgery in the Netherlands [3, 78]. We expect the high-risk group (patients with a VAT $<11 \mathrm{~mL} / \mathrm{kg} / \mathrm{min}$ ) to have a morbidity rate of $50 \%$. Dunne et al. stated that a $10 \%$ improvement in cardiorespiratory fitness would move $30 \%$ of the patients from high to low operative risk [25, 28]. Robinson et al. [34] showed that $21 \%$ of the non-frail colorectal patients had a postoperative complication, versus $40 \%$ in the pre-frail group and $58 \%$ in frail patients. We hypothesized a complication rate of $20 \%$ in the prehabilitation group.

For the sample size calculation we used the computer program PS Power and Sample Size Calculations version 3.0, January 2009 (Copyright $\odot$ 1997-2009 by William D. Dupont and Walton D. Plummer) [79, 80]. Using an alpha of 0.0492 (due to one interim analysis) and a power of 0.80 , we need 39 patients in each group to find statistically significant results. Taking into account $10 \%$ dropout, we need 43 patients in each group.

\section{Procedures for data checking and entering}

Data (coded on study code) will be entered in the Statistical Package for the Social Sciences for Windows (SPSS, version 23.0, IBM, SPSS Inc., Chicago, IL, USA). All data are held in an encrypted format and will be stored in a secured locked room. All variables will be checked for the number of missing, impossible or improbable values, prior to statistical analysis. In case of improbable or impossible values, the patient's data file will be checked by the data manager. Descriptive statistics will be calculated for all variables, and distributional assumptions will be checked. The study coordinator, chief investigator, and a blinded data analyst will have access to the final trial dataset. Due to the low risk nature of the intervention, the Medical Ethics Committee determined that a data monitoring committee is not required.

\section{Statistical analysis}

Nominal variables will be presented as numbers with percentages. Continuous variables will be presented as mean $\pm \mathrm{SD}$, or as median and interquartile range, as appropriate. Data will be presented in tables and figures. All tests will be performed on the intention to treat population, and on the per-protocol population. Data distribution will be checked with help of an epidemiologist.

For categorical variables, chi-squared tests or Fisher's Exact tests, as appropriate, will be performed to analyze the difference between the intervention and control group. For continuous variables this will be done by independent samples t-tests or Mann-Whitney $\mathrm{U}$ tests, as appropriate. A repeated measurements analysis (mixed models in SPSS) will be performed to assess changes over time in continuous variables. A cost-effectiveness analysis will be performed, with the use of quality adjusted life years. Withdrawn patients will be followed by checking the patient's data file. We will use the intention-to-treat analysis to avoid various misleading artifacts.

When half of the patients needed that are calculated (43 patients) are included, a blinded independent data analyst will perform an interim analysis with a stopping rule according to O'Brien-Fleming [81]. If this interim analysis shows a significant difference in postoperative complications between the prehabilitation group and the usual care group (at $P<0.0054$ ), this data analyst will contact the Medical Ethics Committee, and debate the outcome of the interim analysis and its eventual repercussions. Thereupon this committee will advise the principle investigator of the research team (JK) to decide to stop the inclusion in case of superiority. If the interim analysis shows no or minimal difference $(<10 \%$ on overall complications), the study will be stopped due to futility. Because of the interim analysis, the final $P$-value that is considered to be significant is reduced to $P<0.0492$.

\section{Discussion}

Surgery is an important treatment modality in patients with colorectal cancer. Unfortunately, over $30 \%$ of patients develop a postoperative complication after elective colorectal resection [3]. Older patients, especially frail patients, with a poor cardiorespiratory fitness are more prone to postoperative complications and require specific preoperative risk stratification. Optimizing preoperative physical 
fitness (e.g., cardiorespiratory fitness, muscle strength, and functional mobility) through prehabilitation may improve postoperative outcomes in patients undergoing major abdominal surgery [6]. Currently, there is a lack of information in the literature about the effect of preoperative exercise training on overall postoperative complications in high-risk older patients with colorectal cancer. Most of the studies so far were rather underpowered, heterogeneous, and biased toward selection of patients with low risk of postoperative complications $[6,29,30]$. The primary aim of our current randomized controlled trial is to measure the effect of a three-week prehabilitation program on postoperative complications in patients with a poor cardiorespiratory fitness, as indicated by a VAT < $11 \mathrm{~mL} / \mathrm{kg} / \mathrm{min}$, who will undergo elective colorectal resection for colorectal cancer or dysplasia grade I,II, or III.

Postoperative complications can extend length of hospital stay, and may lead to repeated hospital admissions, chronic ill health, and a decrease or even permanent loss in functional capacity and health-related quality of life [6]. Patients with a higher cardiorespiratory fitness might be more resilient to cope with the stress associated with major abdominal surgery, with subsequent better postoperative outcomes. Optimizing preoperative cardiorespiratory fitness may therefore not only decrease postoperative complications, but may also decrease associated hospital costs, enhance the patient's quality of life, and promote independent functioning in daily life. Our intervention fits well with the 'prevention of limitations to function' view in the elderly, of the Health Council of the Netherlands [82], as well as with the recently proposed new schemes of the international classification of functioning, disability, and health (ICF) to promote functioning and health [83].

According to the Dutch guidelines, patients will ideally be operated within five weeks from time of pathologicanatomic diagnosis, and at latest within seven weeks [3]. The period between the decision for surgery and the actual surgery is limited, and therefore the valuable available time for prehabilitation is short. Moreover, some patients experience (severe) complaints of their illness, and therefore resection cannot be postponed for several weeks. However, given the need for prehabilitation interventions to be both effective and time-efficient, Weston et al. [84] indicated that carefully designed and supervised high-intensity interval training programs might be a promising perioperative strategy for enhancing cardiorespiratory fitness within a short period.

The present study, like all studies do, bares some limitations. Firstly, merely patients in the prehabilitation group will perform a second CPET to investigate the effect of the prehabilitation program on cardiorespiratory fitness. Consequently, we are not able to investigate the changes in cardiorespiratory fitness in the preoperative waiting period in the usual care group. However, the waiting period in patients in the usual care group was kept as short as possible to prevent these patients to improve their level of fitness by organizing their own preoperative exercise training program. Moreover, a study in patients planned for elective hepatic resection for colorectal liver metastases reported no significant differences in CPET variables in the control group after a four-week waiting period [25]. Secondly, the prehabilitation program in the present study consists only of a preoperative exercise training program, while it was recently indicated that studies evaluating a prehabilitation program combined with a nutritional intervention before elective major surgery in adults are producing encouraging early results [31]. However, definitive clinical evidence of physical and nutritional prehabilitation is currently very limited $[31,85,86]$. Thirdly, up till now, patient inclusion appears to be challenging. Not all patients are able and/or willing to attend the prehabilitation program due to personal, logistical, and time limitations (e.g., restricted time availability between the first outpatient visit and surgery, living too far away from the community physical therapy practice, and/or no traveling opportunities, planned vacation prior to surgery, not interested in participation). Moreover, at the start of the present study patients in both groups were informed about the possible effects of prehabilitation. We experienced that some patients in the usual care group initiated preoperative physical exercise training themselves, possibly resulting in the risk that we will not be able to detect a difference between the prehabilitation and usual care group. Therefore, we made an amendment to the study protocol (approved in April 2016 by the Medical Ethics Committee Twente) in which patients in the prehabilitation group and the usual care group will be informed differently. The patients in the usual care group will not receive information about the possible effects of prehabilitation and their elective colorectal resection was planned at the earliest convenience. Fourthly, in the present study a geriatric assessment will be completed at baseline only. Baseline data as predictors of postoperative outcome may be confounded by the effect of the prehabilitation program. Therefore, only data from patients in the usual care group will be used to investigate the value of a limited geriatric assessment to perform preoperative risk stratification. However, the power for this secondary objective might be inadequate, as the sample size calculation was not based on this objective.

We believe that preoperative exercise training in patients 'merely' undergoing elective colorectal resection will be only cost-effective and sustainably implementable in usual care pathways when performed in high-risk patients (those with a low cardiorespiratory fitness, e.g., a VAT $<11 \mathrm{~mL} / \mathrm{kg} / \mathrm{min})$. Hence, an adequate 
preoperative risk stratification protocol, such as the protocol used in the current study (a CPET for patients with a VSAQ score $<7$ METs, of which those patients with a VAT $<11 \mathrm{~mL} / \mathrm{kg} / \mathrm{min}$ at the CPET will be eligible for and advised to participate in prehabilitation), will probably lead to a feasible sustainable implementation of the prehabilitation concept. For patients undergoing elective colorectal resection and (neo)adjuvant chemotherapy and/or radiotherapy, prehabilitation might be cost-effective in all patients. Cost-effectiveness should be expected, and therefore evaluated, in terms of, among other things, fewer postoperative complications, fewer reoperations, less intense clinical care, a shorter length of stay, a more effective surgical planning (processoptimization), fewer readmissions, less intense rehabilitation, shorter rehabilitation period, and earlier resumption of work.

The strengths of our present study are the selection of high-risk patients, the clear description of the study design and prehabilitation program, the 1:1 randomization between the prehabilitation and usual care group, the use of validated measurement instruments, and the adjustment of the physical exercise training program to the cardiorespiratory fitness of the individual patient based upon the results of the CPET. Moreover, prehabilitation will be performed in a community physical therapy practice in the catchment of the patient's home. In this way we will try to reach the frailest patients who are not capable to visit the hospital each time.

\section{Additional file}

Additional file 1: Patient Consent Form. (DOCX $12 \mathrm{~kb}$ )

\begin{abstract}
Abbreviations
ASA: American Society of Anesthesiologists; CPET: Cardiopulmonary exercise test; EORTC QLQ-C30: Quality of life questionnaire of the European organization for research and treatment of cancer; EQ-5D: EuroQol 5D; GDS 15: Geriatric Depression Scale 15; GFI: Groningen Frailty Indicator; IADL: Instrumental activities of daily living; MET: Metabolic equivalent of task; SNAQ: Short nutritional assessment questionnaire; TUG: Timed up-and-go; VAT: Ventilatory anaerobic threshold; WHO: World Health Organization
\end{abstract}

\section{Acknowledgements}

Not applicable.

\section{Trial status}

The trial started patient inclusion in February 2014, and is expected to be completed by the end of 2018 .

\section{Funding}

Amgen will partly fund this study. Amgen had no role in the design of the study, and will not have a role in collection, analysis, and interpretation of data and in writing the manuscript.

\section{Availability of data and materials}

Not applicable at this stage. The datasets that will be used and/or analyzed during the current study will be available from the corresponding author (a.berkel@mst.nl) on reasonable request.

\section{Authors' contributions}

The chief investigator (JMK) conceived the study, and has overall conduct of the study. The study coordinator (AEB) ensures day-to-day management of the study. AEB, BCB, MSK, HK, PW, FHJ, MME, ANW, MB, JP, MJD, NLM, and $J M K$ contributed to the study design. $A E B$ and $B C B$ drafted the manuscript, which underwent revision by all other authors. All authors read and approved the final manuscript.

\section{Authors' information}

Not applicable.

\section{Ethics approval and consent to participate}

Medical ethics approval was granted by the Medical Ethics Committee Twente, Enschede, the Netherlands (P13-18). The study is registered in www.trialregister.nl, trial registration number NTR4032. Written informed consent will be obtained from all patients before enrolment in the study.

Consent for publication

Not applicable.

\section{Competing interests}

The study has received funding from a commercial organization (Amgen).

\section{Publisher's Note}

Springer Nature remains neutral with regard to jurisdictional claims in published maps and institutional affiliations.

\section{Author details}

'Department of Surgery, Medisch Spectrum Twente, PO Box 50 000, 7500, KA, Enschede, The Netherlands. ${ }^{2}$ Department of Epidemiology, Faculty of Health, Medicine and Life Sciences, Care and Public Health Research Institute (CAPHRI), Maastricht University, PO Box 616, 6200, MD, Maastricht, The Netherlands. ${ }^{3}$ Physical therapy practice, Fysio Twente, J.J. van Deinselaan 34a, 7541, PE, Enschede, The Netherlands. ${ }^{4}$ Physical therapy practice, FITclinic, Roomweg 180, 7523, BT, Enschede, The Netherlands. ${ }^{5}$ Department of Pulmonology, Medisch Spectrum Twente, PO Box 50 000, 7500, KA, Enschede, The Netherlands. ${ }^{6}$ Department of Internal medicine, Medisch Spectrum Twente, PO Box 50 000, 7500, KA, Enschede, The Netherlands. ${ }^{7}$ Department of Gastroenterology and Hepatology, Medisch Spectrum Twente, PO Box 50 000, 7500, KA, Enschede, The Netherlands. ${ }^{8}$ Epidemiology, Medisch Spectrum Twente, PO Box 50 000, 7500, KA, Enschede, The Netherlands. ${ }^{9}$ Department of Surgery, Ziekenhuisgroep Twente, PO Box 7600, 7600, SZ, Almelo, The Netherlands. ${ }^{10}$ Top Sector Life Sciences and Health (Health Holland), Laan van Nieuw Oost-Indië 334, 2593, CE, The Hague, The Netherlands.

Received: 26 April 2017 Accepted: 28 January 2018

Published online: 21 February 2018

\section{References}

1. Haggar FA, Boushey RP. Colorectal cancer epidemiology: incidence, mortality, survival, and risk factors. Clin Colon Rectal Surg. 2009;22(4):191-7.

2. Integraal Kankercentrum Nederland (IKNL). Ciifers over kanker. 2016. Available at: https://www.cijfersoverkanker.nl/. Accessed 10 Nov 2016.

3. Dutch Institute for Clinical Auditing (DICA) Leiden. DICA jaarrapportage. Dutch Surgical Colorectal Audit. 2014. Available at: https:/dica.nl/ jaarrapportage-2014/. Accessed 12 Dec 2017

4. Hulzebos EH, van Meeteren NL. Making the elderly fit for surgery. Br J Surg. 2016;103(4):463.

5. Dutch Institute for Clinical Auditing (DICA) Leiden. DICA jaarrapportage. Dutch Surgical Colorectal Audit. 2016. Available at: http://dica.nl/ jaarrapportage-2016/dsca. Accessed 12 Dec 2017.

6. Moran J, Guinan E, Mc Cormick P, Larkin J, Mockler D, Hussey J, et al. The ability of prehabilitation to influence postoperative outcome after intraabdominal operation: a systematic review and meta-analysis. Surgery. 2016; 160(5):1189-201.

7. Sinclair RC, Danjoux GR, Goodridge V, Batterham AM. Determination of the anaerobic threshold in the pre-operative assessment clinic: inter-observer measurement error. Anaesthesia. 2009;64(11):1192-5. 
8. Kothmann E, Danjoux G, Owen SJ, Parry A, Turley AJ, Batterham AM. Reliability of the anaerobic threshold in cardiopulmonary exercise testing of patients with abdominal aortic aneurysms. Anaesthesia. 2009;64(1):9-13.

9. Older $\mathrm{P}, \mathrm{Hall}$ A. Clinical review: how to identify high-risk surgical patients. Crit Care. 2004:8(5):369-72.

10. Junejo MA, Mason JM, Sheen AJ, Moore J, Foster P, Atkinson D, et al. Cardiopulmonary exercise testing for preoperative risk assessment before hepatic resection. Br J Surg. 2012;99(8):1097-104.

11. Ausania F, Snowden CP, Prentis JM, Holmes LR, Jaques BC, White SA, et al. Effects of low cardiopulmonary reserve on pancreatic leak following pancreaticoduodenectomy. Br J Surg. 2012;99(9):1290-4.

12. Hightower CE, Riedel BJ, Feig BW, Morris GS, Ensor JE Jr, Woodruff VD, et al. A pilot study evaluating predictors of postoperative outcomes after major abdominal surgery: physiological capacity compared with the ASA physical status classification system. Br J Anaesth. 2010;104(4):465-71.

13. Hartley RA, Pichel AC, Grant SW, Hickey GL, Lancaster PS, Wisely NA, et al. Preoperative cardiopulmonary exercise testing and risk of early mortality following abdominal aortic aneurysm repair. Br J Surg. 2012;99(11):1539-46.

14. Snowden CP, Prentis JM, Anderson HL, Roberts DR, Randles D, Renton M, et al. Submaximal cardiopulmonary exercise testing predicts complications and hospital length of stay in patients undergoing major elective surgery. Ann Surg. 2010;251(3):535-41

15. Prentis JM, Manas DM, Trenell MI, Hudson M, Jones DJ, Snowden CP. Submaximal cardiopulmonary exercise testing predicts 90-day survival after liver transplantation. Liver Transpl. 2012;18(2):152-9.

16. Prentis JM, Trenell MI, Jones DJ, Lees T, Clarke M, Snowden CP. Submaximal exercise testing predicts perioperative hospitalization after aortic aneurysm repair. J Vasc Surg. 2012;56(6):1564-70

17. Wilson RJ, Davies S, Yates D, Redman J, Stone M. Impaired functional capacity is associated with all-cause mortality after major elective intraabdominal surgery. Br J Anaesth. 2010;105(3):297-303.

18. Hennis PJ, Meale PM, Hurst RA, O'Doherty AF, Otto J, Kuper M, et al. Cardiopulmonary exercise testing predicts postoperative outcome in patients undergoing gastric bypass surgery. Br J Anaesth. 2012;109(4):566-71.

19. Older P, Hall A, Hader R. Cardiopulmonary exercise testing as a screening test for perioperative management of major surgery in the elderly. Chest. 1999;116(2):355-62.

20. Smith TB, Stonell C, Purkayastha S, Paraskevas P. Cardiopulmonary exercise testing as a risk assessment method in non cardio-pulmonary surgery: a systematic review. Anaesthesia. 2009;64(8):883-93.

21. Chandrabalan W, McMillan DC, Carter R, Kinsella J, McKay CJ, Carter CR, et al. Pre-operative cardiopulmonary exercise testing predicts adverse postoperative events and non-progression to adjuvant therapy after major pancreatic surgery. HPB (Oxford). 2013;15(11):899-907.

22. Older P, Smith R, Courtney P, Hone R. Preoperative evaluation of cardiac failure and ischemia in elderly patients by cardiopulmonary exercise testing. Chest. 1993:104(3):701-4.

23. O'Doherty AF, West M, Jack S, Grocott MP. Preoperative aerobic exercise training in elective intra-cavity surgery: a systematic review. $\mathrm{Br} J$ Anaesth. 2013;110(5):679-89.

24. Barberan-Garcia A, Ubre M, Roca J, Lacy AM, Burgos F, Risco R, et al. Personalised prehabilitation in high-risk patients undergoing elective major abdominal surgery: a randomized blinded controlled trial. Ann Surg. 2018; 267(1):50-6.

25. Dunne DF, Jack S, Jones RP, Jones L, Lythgoe DT, Malik HZ, et al. Randomized clinical trial of prehabilitation before planned liver resection. $\mathrm{Br}$ J Surg. 2016;103(5):504-12.

26. Jack S, West M, Grocott MP. Perioperative exercise training in elderly subjects. Best Pract Res Clin Anaesthesiol. 2011;25(3):461-72.

27. Snowden CP, Prentis J, Jacques B, Anderson H, Manas D, Jones D, et al. Cardiorespiratory fitness predicts mortality and hospital length of stay after major elective surgery in older people. Ann Surg. 2013; 257(6):999-1004.

28. Dunne DF, Jones RP, Poston GJ, Malik HZ, Palmer DH, Jack S, et al. A prehabilitation program for liver surgery. HPB. 2012;14(Suppl. 2):107-287.

29. Bruns ER, Van den Heuvel B, Buskens CJ, Van Duijvendijk P, Festen S, Wassenaar EB, et al. The effects of physical prehabilitation in elderly patients undergoing colorectal surgery: a systematic review. Color Dis. 2016;18(8):0267-77.

30. Hijazi Y, Gondal U, Aziz O. A systematic review of prehabilitation programs in abdominal cancer surgery. Int J Surg. 2017;39:156-62.
31. West MA, Wischmeyer PE, Grocott MP. Prehabilitation and nutritional support to improve perioperative outcomes. Curr Anesthesiol Rep. 2017;7(4):340-9.

32. Hoogeboom TJ, Oosting E, Vriezekolk JE, Veenhof C, Siemonsma PC, de Bie RA, et al. Therapeutic validity and effectiveness of preoperative exercise on functional recovery after joint replacement: a systematic review and metaanalysis. PLoS One. 2012;7(5):e38031.

33. Pouwels S, Hageman D, Gommans LN, Willigendael EM, Nienhuijs SW, Scheltinga MR, et al. Preoperative exercise therapy in surgical care: a scoping review. J Clin Anesth. 2016;33:476-90.

34. Robinson TN, Wu DS, Pointer L, Dunn CL, Cleveland JC Jr, Moss M. Simple frailty score predicts postoperative complications across surgical specialties. Am J Surg. 2013;206(4):544-50.

35. Myers J, Do D, Herbert W, Ribis P, Froelicher VF. A nomogram to predict exercise capacity from a specific activity questionnaire and clinical data. Am J Cardiol. 1994:73(8):591-6.

36. Ainsworth BE, Haskell WL, Leon AS, Jacobs DR Jr, Montoye HJ, Sallis JF, et al. Compendium of physical activities: classification of energy costs of human physical activities. Med Sci Sports Exerc. 1993;25(1):71-80.

37. Press WH, Teukolsky S, Vetterling WT, Flannery BP. Numerical recipes in C: the art of scientific computing. New York: Cambridge University Press; 1992

38. Gibala MJ, Jones AM. Physiological and performance adaptations to highintensity interval training. Nestle Nutr Inst Workshop Ser. 2013;76:51-60.

39. Gibala MJ, Little JP, Macdonald MJ, Hawley JA. Physiological adaptations to low-volume, high-intensity interval training in health and disease. J Physiol. 2012;590(5):1077-84

40. de Vreede PL, Samson MM, Van Meeteren NL, Duursma SA, Verhaar HJ. Functional-task exercise versus resistance strength exercise to improve daily function in older women: a randomized, controlled trial. J Am Geriatr Soc. 2005;53(1):2-10

41. Punt IM, Bongers BC, Van Beijsterveld CA, Hulzebos HJ, Dronkers JJ, Van Meeteren NL. Surgery: moving people, improving outcomes. In: Geriatrics. Hyderabad: Avid Science; 2016. p. 2-30

42. American Thoracic Society. American College of Chest Physicians. ATS/ACCP statement on cardiopulmonary exercise testing. Am J Respir Crit Care Med. 2003;167(2):211-77.

43. Wasserman K, Hansen JE, Sue DY, Stringer WW, Whipp BJ. Principles of exercise testing and interpretation. Including pathophysiology and clinical applications (4th edition). Philadelphia: Lippincott Williams \& Wilkins; 2005.

44. Bongers BC, Berkel AE, Klaase JM, Van Meeteren NL. An evaluation of the validity of the pre-operative oxygen uptake efficiency slope as an indicator of cardiorespiratory fitness in elderly patients scheduled for major colorectal surgery. Anaesthesia. 2017;72(10):1206-16.

45. Beaver WL, Wasserman K, Whipp BJ. A new method for detecting anaerobic threshold by gas exchange. J Appl Physiol (1985). 1986;60(6):2020-7.

46. Borley NR, Achan V. Respiratory physiology. In: Instant physiology. Hoboken: Wiley-Blackwell; 2000.

47. DeCato TW, Bradley SM, Wilson EL, Hegewald MJ. Repeatability and meaningful change of CPET parameters in normal subjects. Med Sci Sports Exerc. 2017. https://doi.org/10.1249/MSS.0000000000001474. Epub ahead of print.

48. Mathiowetz V. Comparison of Rolyan and Jamar dynamometers for measuring grip strength. Occup Ther Int. 2002;9(3):201-9.

49. Mahalakshmi VN, Ananthakrishnan N, Kate V, Sahai A, Trakroo M. Handgrip strength and endurance as a predictor of postoperative morbidity in surgical patients: can it serve as a simple bedside test? Int Surg. 2004;89(2):115-21.

50. Kerr A, Syddall HE, Cooper C, Turner GF, Briggs RS, Sayer AA. Does admission grip strength predict length of stay in hospitalised older patients? Age Ageing. 2006:35(1):82-4.

51. Martin HJ, Yule V, Syddall HE, Dennison EM, Cooper C, Aihie Sayer A. Is hand-held dynamometry useful for the measurement of quadriceps strength in older people? A comparison with the gold standard Bodex dynamometry. Gerontology. 2006;52(3):154-9.

52. Podsiadlo D, Richardson S. The timed "up \& go": a test of basic functional mobility for frail elderly persons. J Am Geriatr Soc. 1991;39(2):142-8.

53. Mathias S, Nayak US, Isaacs B. Balance in elderly patients: the "get-up and go" test. Arch Phys Med Rehabil. 1986;67(6):387-9.

54. Robinson TN, Wallace Jl, Wu DS, Wiktor A, Pointer LF, Pfister SM, et al. Accumulated frailty characteristics predict postoperative discharge institutionalization in the geriatric patient. J Am Coll Surg. 2011;213(1): 37,42. discussion 42-4

55. Huisman MG, van Leeuwen BL, Ugolini G, Montroni I, Spiliotis J, Stabilini C, et al. "Timed up \& go": a screening tool for predicting 30-day morbidity in 
onco-geriatric surgical patients? A multicenter cohort study. PLoS One 2014;9(1):e86863

56. Kruizenga HM, Seidell JC, de Vet HC, Wierdsma NJ, van Bokhorst-de van der Schueren MA. Development and validation of a hospital screening tool for malnutrition: the short nutritional assessment questionnaire (SNAQ). Clin Nutr. 2005;24(1):75-82.

57. Oken MM, Creech RH, Tormey DC, Horton J, Davis TE, McFadden ET, et al. Toxicity and response criteria of the eastern cooperative oncology group. Am J Clin Oncol. 1982;5(6):649-55.

58. Dripps RD, Lamong A, Eckenhoff JE. The role of anesthesia in surgical mortality. JAMA. 1961;178:261-6.

59. Charlson ME, Pompei $P$, Ales $K L$, MacKenzie $C R$. A new method of classifying prognostic comorbidity in longitudinal studies: development and validation. J Chronic Dis. 1987;40(5):373-83.

60. Barnett S, Moonesinghe SR. Clinical risk scores to guide perioperative management. Postgrad Med J. 2011;87(1030):535-41.

61. Ouellette JR, Small DG, Termuhlen PM. Evaluation of Charlson-age Comorbidity index as predictor of morbidity and mortality in patients with colorectal carcinoma. J Gastrointest Surg. 2004:8(8):1061-7.

62. Lawton MP, Brody EM. Assessment of older people: self-maintaining and instrumental activities of daily living. Gerontologist. 1969:9(3):179-86.

63. Lawton MP, Moss M, Fulcomer M, Kleban MH. A research and service oriented multilevel assessment instrument. J Gerontol. 1982;37(1):91-9.

64. Lawton MP. Scales to measure competence in everyday activities. Psychopharmacol Bull. 1988;24(4):609-14.

65. Sheikh JI, Yesavage JA. Geriatric depression scale (GDS): recent evidence and development of a shorter version. Clin Gerontol. 1986;5:165-73.

66. Yesavage JA, Brink TL, Rose $\mathrm{TL}$, Lum $\mathrm{O}$, Huang $\mathrm{V}$, Adey $\mathrm{M}$, et al. Development and validation of a geriatric depression screening scale: a preliminary report. J Psychiatr Res. 1982;17(1):37-49.

67. Aaronson NK, Ahmedzai S, Bergman B, Bullinger M, Cull A, Duez NJ, et al. The European Organization for Research and Treatment of cancer QLQ-C30: a quality-of-life instrument for use in international clinical trials in oncology. J Natl Cancer Inst. 1993;85(5):365-76.

68. Fayers P, Bottomley A. EORTC quality of life group, quality of life unit. Quality of life research within the EORTC-the EORTC QLQ-C30. European Organisation for Research and Treatment of Cancer. Eur J Cancer. 2002;38(Suppl 4):S125-33.

69. Schuurmans $H$, Steverink N, Lindenberg S, Frieswijk N, Slaets JP. Old or frail: what tells us more? J Gerontol A Biol Sci Med Sci. 2004;59(9):M962-5.

70. McDowell I, Newell C. Measuring health: a guide to rating scales and questionnaires (2nd edition). New York: Oxford University Press; 1996.

71. de Raaij EJ, Schroder C, Maissan FJ, Pool JJ, Wittink H. Cross-cultural adaptation and measurement properties of the brief illness perception questionnaire-Dutch language version. Man Ther. 2012;17(4):330-5.

72. EuroQol Group. EuroQol-a new facility for the measurement of healthrelated quality of life. Health Policy. 1990;16(3):199-208,

73. Dowson HM, Ballard K, Gage H, Jackson D, Williams P, Rockall TA. Quality of life in the first 6 weeks following laparoscopic and open colorectal surgery. value Health. 2013;16(2):367-72.

74. Schwenkglenks M, Matter-Walstra K. Is the EQ-5D suitable for use in oncology? An overview of the literature and recent developments. Expert Rev Pharmacoecon Outcomes Res. 2016;16(2):207-19.

75. Clavien PA, Barkun J, de Oliveira ML, Vauthey JN, Dindo D, Schulick RD, et al. The Clavien-Dindo classification of surgical complications: five-year experience. Ann Surg. 2009;250(2):187-96.

76. Dindo D, Demartines N, Clavien PA. Classification of surgical complications: a new proposal with evaluation in a cohort of 6336 patients and results of a survey. Ann Surg. 2004;240(2):205-13.

77. Daabiss M. American Society of Anaesthesiologists physical status classification. Indian J Anaesth. 2011;55(2):111-5.

78. Grossmann I, Klaase JM, Avenarius JK, de Hingh $\mathrm{H}_{\text {, Mastboom WJ, Wiggers }}$ $\mathrm{T}$. The strengths and limitations of routine staging before treatment with abdominal CT in colorectal cancer. BMC Cancer. 2011;11:433.

79. Dupont WD, Plummer WD Jr. Power and sample size calculations for studies involving linear regression. Control Clin Trials. 1998;19(6):589-601.

80. Dupont WD, Plummer WD Jr. Power and sample size calculations. A review and computer program. Control Clin Trials. 1990;11(2):116-28.

81. O'Brien PC, Fleming TR. A multiple testing procedure for clinical trials. Biometrics. 1979;35(3):549-56.

82. Health Council of the Netherlands. Prevention in the elderly: Focus on functioning in daily life. The Hague: Health Council of the Netherlands,
2009; publication no. 2009/07. Available at https://www.gezondheidsraad.nl/ sites/default/files/200907r.pdf. Accessed 10 Mar 2017.

83. Heerkens YF, De Weerd M, Huber M, De Brouwer CP, Van Der Veen S, Perenboom RJ, et al. Reconsideration of the scheme of the international classification of functioning, disability and health: incentives from the Netherlands for a global debate. Disabil Rehabil. 2017:1-9.

84. Weston M, Weston $\mathrm{KL}$, Prentis JM, Snowden CP. High-intensity interval training (HIT) for effective and time-efficient pre-surgical exercise interventions. Perioper Med (Lond). 2016;5:2. https://doi.org/10.1186/s13741015-0026-8. eCollection 2016

85. Bruns ER, Argillander TE, Van Den Heuvel B, Buskens CJ, Van Duijvendijk P Winkels RM, et al. Oral nutrition as a form of pre-operative enhancement in patients undergoing surgery for colorectal cancer: a systematic review. Surg Infect. 2018;19(1):1-10.

86. Looijaard SM, Slee-Valentijn MS, Otten RH, Maier AB. Physical and nutritional prehabilitation in older patients with colorectal carcinoma: a systematic review. J Geriatr Phys Ther. 2017. https://doi.org/10.1519/JPT. 0000000000000125 . Epub ahead of print.

\section{Submit your next manuscript to BioMed Central and we will help you at every step:}

- We accept pre-submission inquiries

- Our selector tool helps you to find the most relevant journal

- We provide round the clock customer support

- Convenient online submission

- Thorough peer review

- Inclusion in PubMed and all major indexing services

- Maximum visibility for your research

Submit your manuscript at www.biomedcentral.com/submit
) Biomed Central 